Derivation and Solution of Multifrequency Radiation Diffusion Equations for Homogeneous Refractive Lossy Media

A. I. Shestakov, R. M. Vignes, J. S. Stolken

January 11,2010

Journal of Computational Physics 
This document was prepared as an account of work sponsored by an agency of the United States government. Neither the United States government nor Lawrence Livermore National Security, LLC, nor any of their employees makes any warranty, expressed or implied, or assumes any legal liability or responsibility for the accuracy, completeness, or usefulness of any information, apparatus, product, or process disclosed, or represents that its use would not infringe privately owned rights. Reference herein to any specific commercial product, process, or service by trade name, trademark, manufacturer, or otherwise does not necessarily constitute or imply its endorsement, recommendation, or favoring by the United States government or Lawrence Livermore National Security, LLC. The views and opinions of authors expressed herein do not necessarily state or reflect those of the United States government or Lawrence Livermore National Security, LLC, and shall not be used for advertising or product endorsement purposes. 


\title{
Derivation and solution of multifrequency radiation diffusion equations for homogeneous refractive lossy media*
}

\author{
A. I. Shestakov, R. M. Vignes and J. S. Stölken \\ Lawrence Livermore National Laboratory \\ Livermore CA 94550
}

January 12, 2010

\begin{abstract}
Starting from the radiation transport equation for homogeneous, refractive lossy media, we derive the corresponding time-dependent multifrequency diffusion equations. Zeroth and first moments of the transport equation couple the energy density, flux and pressure tensor. The system is closed by neglecting the temporal derivative of the flux and replacing the pressure tensor by its diagonal analogue. The system is coupled to a diffusion equation for the matter temperature. We are interested in modeling annealing of silica $\left(\mathrm{SiO}_{2}\right)$. We derive boundary conditions at a planar air-silica interface taking account of reflectivities. The spectral dimension is discretized into a finite number of intervals leading to a system of multigroup diffusion equations. Three simulations are presented. One models cooling of a silica slab, initially at $2500{ }^{\circ} \mathrm{K}$, for $10 \mathrm{~s}$. The other two are $1 \mathrm{D}$ and $2 \mathrm{D}$ simulations of irradiating silica with a $\mathrm{CO}_{2}$ laser, $\lambda=10.59 \mu \mathrm{m}$. In $2 \mathrm{D}$, we anneal a disk (radius $=0.4$, thickness $=0.4 \mathrm{~cm}$ ) with a laser, Gaussian profile ( $r_{0}=0.5 \mathrm{~mm}$ for $1 / e$ decay.)
\end{abstract}

\section{Introduction}

This paper derives a tractable system of equations to model radiation effects when annealing a refractive, absorbing medium. For glass, the importance of radiation as an effective heat transfer mechanism has long been recognized. Gardon [7] and Condon [5] present valuable surveys of the process. Early work modeled the enhanced energy transfer due to radiation by adding to the conventional energy flux $k_{m} \nabla T$ a gray radiative heat flux proportional to $n^{2} \sigma T^{4}$, where $n$

${ }^{*}$ This work performed under the auspices of the U.S. Department of Energy by Lawrence Livermore National Laboratory under Contract DE-AC52-07NA27344. 
is the medium's (average) refractive index and $\sigma$ is the Stefan-Boltzmann constant. This is the "single temperature" model in which the radiation field is assumed to be tightly coupled to the medium. Hence, the radiation spectrum is given by the matter emission source $n^{2} \mathcal{B}_{\nu}(T)$, where $\mathcal{B}_{\nu}(T)$ is the Planck function. However, for glass in general, and silica $\left(\mathrm{SiO}_{2}\right)$ in particular, the absorptivity has a complex spectral structure. At room temperature, in the IRvisible part of the spectrum $(\lambda \approx 1 \mu \mathrm{m})$ the medium is transparent; the opacity $\kappa \approx 0.01 \mathrm{~cm}^{-1}$. However, for select longer wavelengths, it is quite opaque; at $\lambda \approx 10.6 \mu \mathrm{m}, \kappa>10^{4}$, an increase of six orders of magnitude. Hence, we should not expect tight coupling and, as we show below, require a separate equation for heat (energy) transfer due to radiation. At a minimum, one could average the radiation intensity over both direction of propagation and frequency thereby deriving an equation for a radiation temperature $T_{r}$. We define $T_{r}$ in Section 6. However, modeling radiation with only a scalar equation can yield erroneous results. A single $T_{r}$ equation is the limiting case of a multigroup formulation. The latter arises when the frequency variable is discretized into a finite number of groups. Our results show that the quality of the result degrades as we coarsen the frequency discretization.

The subject of coupling radiation transport to a heat conduction equation (HC) has been discussed by many authors. We were initially guided by Larsen et al [11] and [12], work in which the authors start by adding the steady-state radiative transfer equation to $\mathrm{HC}$. The gist of the Larsen et al papers applies the "simplified $P_{n}$ " $\left(S P_{n}\right)$ approximation, developed for the multigroup neutron transport equation, to glass annealing. (Larsen et al [13] present a theoretical foundation of $S P_{n}$.) In the 2002 paper, Larsen et al [11] develop a frequency averaged model for the radiation field. They define a dimensionless parameter $\epsilon \doteq\left(\kappa_{\text {ref }} x_{\text {ref }}\right)^{-1}$, where $\kappa_{\text {ref }}$ and $x_{\text {ref }}$ are reference absorption and length scales. The parameter $\epsilon$ is used for an asymptotic expansion in which, by keeping successively more terms (powers of $\epsilon$ ), one obtains the $S P_{1}, S P_{2}$, etc. (frequency averaged) equations. The $S P_{1}$ system is equivalent to gray radiation diffusion with a Rosseland averaged opacity; radiation is defined by one scalar field. In theory, the $S P_{2}$ system, which models radiation with two scalar fields, provides a correction to diffusion.

In the 2003 paper, Larsen et al [12] include frequency dependence and derive a system similar to standard multigroup diffusion. Their $S P_{2}$ formulation defines the radiation field in terms of a single, frequency-dependent variable $\phi$. However, we note an error; $\phi$ is not the scalar flux, as defined in [12], but instead a linear combination of the flux and emission source that in the 2002 paper is denoted by $\xi$ [11]. The 2003 paper has another, less important error (or misprint) in the boundary condition for the temperature. We clarify both issues below.

We admit to reservations about the applicability of the $S P_{n}$ approximation to model heat transport in glass. First, the Larsen et al derivations [11] and [12] hinge on having an optically thick domain, i.e., small $\epsilon$. But, glass is 
not optically thick, at least not at room temperature over the optical band. As the medium cools, this interval contributes significantly to heat loss. So at least for this band, $\epsilon$ is large, not small, since the mean free path $\kappa_{\text {ref }}^{-1}$ is significantly longer than the medium's dimension $x_{\text {ref }}$. However, in fairness, we note that at least for a sufficiently fine frequency discretization, the Larsen et al multifrequency $S P_{2}$ equations can be derived by expanding the steady state radiation intensity equation in terms of Legendre polynomials and discarding $P_{3}$ and higher terms, Shestakov [20]. Another reservation is the ambiguous nature of Larsen et al's fundamental unknown $W_{i}$. It is an integral over frequency intervals of $\xi$ divided by the opacity, where $\xi$ is the linear combination described above. A third reservation, albeit of an aesthetic nature, is that the Larsen et al system requires a fair amount of scrutiny before one finds the exchange term between the medium's internal energy and radiation. Lastly, the $S P_{2}$ system stems from neglecting the temporal derivative of radiation. We discuss the implications in Section 7. However, we note that keeping the term, as we do, leads to conventional multigroup diffusion. By ignoring moments of the reflectivity and setting the refractive index to unity, we recover the equations found in standard radiation-hydrodynamic codes.

We now summarize our paper. In the next Section we derive the multigroup equations. Boundary conditions are discussed in Section 3. We first consider the radiation field. Following Larsen et al [11], arrive at a "mixed-type" condition that includes moments of the reflectivity. Setting the moments to zero reduces to the familiar Milne boundary condition, viz., a linear relation for the field, its gradient and an external source. The boundary condition for the temperature is derived in Section 3.2. As in [11], we allow for convective heat loss and an "opaque frequency interval" $\left(0, \nu_{0}\right)$, where the opacity is so high that matter radiates as a black body. The condition contains a moment of the reflectivity. The numerical method, a variant of the "partial temperature scheme" of Lund and Wilson [14], is developed in Section 4. Section 5 describes the material properties (absorption, refractive indexes, opacities, reflectivity moments) used in our simulations. Numerical results appear in Section 6. In Section 6.1, we present 1D simulations that model the cooling of a $1 \mathrm{~cm}$ thick slab of $\mathrm{SiO}_{2}$, initially at $T=2500$. We present convergence with group number and mesh size. Also, we compare results of our equations with those from conventional multigroup diffusion (no reflectivity, no refraction). Section 6.2 describes simulations of $\mathrm{SiO}_{2}$, initially at room temperature, irradiated by a $\mathrm{CO}_{2}$ laser. For the 1D simulation, we model an infinite slab irradiated for $1 \mathrm{~s}$ at power 4.34 $\mathrm{W} / \mathrm{mm}^{2}$. In $2 \mathrm{D}$, we model a disk heated axially, for $10 \mathrm{~s}$, by a $10 \mathrm{~W}$ laser with a 1/e diameter of $1 \mathrm{~mm}$. Concluding remarks appear in Section 7 . 


\section{Derivation of equations}

We derive a tractable system of equations to model radiation effects when annealing a refractive, absorbing medium. In our applications, the temperature is raised by a laser that may be modeled as an external, temporally and spatially varying energy source. Energy deposition can be very local, for a $\mathrm{CO}_{2}$ laser operating at wavelength $\lambda=10.6 \mu \mathrm{m}$, the absorption depth, which varies with temperature $T$, can be as small as $5 \mu \mathrm{m}$, [15]. Depending on the laser power, the deposition raises the local temperature from $T=298$ to $2500{ }^{\circ} \mathrm{K}$. Heat is carried away by several processes: conduction via Fourier's law, radiation emission and convection into the surrounding air. Radiation has its own mechanisms for energy dissipation: transport and losses at the interface.

In a material reference frame and ignoring material motion, two equations model the process. The first governs material internal energy flow, i.e., heat conduction,

$$
C \partial_{t} T=\nabla \cdot k_{m} \nabla T+S-K_{r, m} .
$$

In Eq. (1), $C\left(\mathrm{erg} / \mathrm{cm}^{3}{ }^{\circ} \mathrm{K}\right)$ is the heat capacity, $k_{m}\left(\mathrm{erg} / \mathrm{cm} \mathrm{sec}^{\circ} \mathrm{K}\right)$ the conductivity, $S\left(\mathrm{erg} / \mathrm{cm}^{3} \mathrm{sec}\right.$ ) an external source (e.g., a laser) and $K_{r, m}$ is the rate of energy exchange between radiation and the medium; it's sign depends on whether locally the radiation is "hotter" than $T{ }^{1}$

The second equation describes radiation energy transport and energy exchange with the medium. For homogeneous ${ }^{2}$, refractive lossy media, we begin with the equation derived by Pomraning [17],

$$
n^{2}\left[\left(1 / v_{g}\right) \partial_{t}\left(I / n^{2}\right)+\boldsymbol{\Omega} \cdot \nabla\left(I / n^{2}\right)\right]=\kappa\left[n^{2} \mathcal{B}_{\nu}(T)-I\right] .
$$

The LS of Eq.(2) differs from Pomraning's Eq.(5.64) et seq. We neglect his last three terms because we ignore spatial and temporal changes of $n$, the refractive index. ${ }^{3}$ In Eq. (2), the fundamental variable, the intensity $I\left(\mathrm{erg} / \mathrm{cm}^{3} \mathrm{sec} \mathrm{Hz} \mathrm{sr}\right)$, depends on time, position $\mathbf{x}$, propagation direction $\boldsymbol{\Omega}$, and frequency $\nu$. Equation (2) introduces the dimensionless refractive index $n$, the group speed $v_{g}$ $(\mathrm{cm} / \mathrm{sec})$, the opacity $\kappa\left(\mathrm{cm}^{-1}\right)$ and the Planck function,

$$
\mathcal{B}_{\nu}(T)=\frac{2 h_{P} \nu^{3} / c^{2}}{\exp \left(h_{P} \nu / k_{B} T\right)-1},
$$

where $h_{P}$ and $k_{B}$ are the Planck and Boltzmann constants, resp. Note that in refractive media, radiation emission is given by $n^{2} \mathcal{B}_{\nu}$ and radiation propagates at the frequency-dependent group speed $v_{g}$, which is related to the phase speed

\footnotetext{
${ }^{1} \mathrm{~A}$ radiation temperature $T_{r}$ is defined in Section 6 .

${ }^{2}$ In a homogeneous medium, the index of refraction and opacity are independent of space and time.

${ }^{3}$ One of the neglected terms has $d \boldsymbol{\Omega} / d s$, which depends on $\nabla n$ ( $s$ is the path length). Another term has $d \nu / d s$, but it varies with $\partial_{t} n$. The third term explicitly contains $\partial_{t} n$.
} 
$v_{p}$, Born \& Wolf [1],

$$
v_{g}=v_{p}-\lambda \frac{d v_{p}}{d \lambda}=v_{p} /\left(1+\frac{\nu}{n} \frac{d n}{d \nu}\right), v_{p}=c / n,
$$

where $\lambda$ is the wavelength and $c(\mathrm{~cm} / \mathrm{sec})$ is the vacuum light speed.

To proceed, we take moments of Eq.(2). For the zeroth moment, we integrate over $\boldsymbol{\Omega}$; for the first moment, we multiply by $\boldsymbol{\Omega}$ before integrating. After defining the spectral energy density $E$, the flux $\mathbf{F}$, and the pressure tensor $\overline{\overline{\mathrm{P}}}$,

$$
\begin{aligned}
E & =\left(1 / v_{g}\right) \int_{4 \pi} d \omega I \\
\mathbf{F} & =\int_{4 \pi} d \omega \Omega I \\
\overline{\overline{\mathrm{P}}} & =\left(1 / v_{g}\right) \int_{4 \pi} d \omega \boldsymbol{\Omega} \boldsymbol{\Omega} I,
\end{aligned}
$$

we obtain,

$$
\begin{aligned}
\partial_{t} E+\nabla \cdot \mathbf{F} & =\kappa\left[4 \pi n^{2} \mathcal{B}_{\nu}(T)-v_{g} E\right] \\
\left(1 / v_{g}\right) \partial_{t}\left(\mathbf{F} / n^{2}\right)+\nabla \cdot\left(v_{g} \overline{\overline{\mathrm{P}}} / n^{2}\right) & =-\kappa \mathbf{F} / n^{2}
\end{aligned}
$$

This gives two equations for three unknowns, $E, \mathbf{F}$ and $\overline{\overline{\mathrm{P}}}$. To close the system, we neglect the temporal derivative in Eq.(5). The reasoning is that

$$
\left(1 / v_{g}\right) \partial_{t}\left(\mathbf{F} / n^{2}\right) \sim \mathbf{F} /\left(v_{g} \tau n^{2}\right),
$$

where $\tau$ is a characteristic time. Comparing this to the RS of Eq.(5), we can drop the temporal derivative as long as $1 \ll v_{g} \tau \kappa$. The speed $v_{g}=\mathcal{O}(c)$, while $\kappa$ is the inverse mean free path. Hence, neglecting the temporal derivative in Eq.(5) means the distance a photon could travel in the absence of absorption is considerably longer that it would.

After the time derivative of Eq.(5) is dropped, we obtain a steady-state condition for the flux in terms of the pressure tensor. We then follow the usual argument (Castor [3], p.73-74) that replaces $\overline{\overline{\mathrm{P}}}$ with a third of the energy density:

$$
\mathbf{F}=-\frac{n^{2}}{\kappa} \nabla \cdot\left(\frac{v_{g}}{n^{2}} \overline{\overline{\mathrm{P}}}\right)=-\frac{n^{2}}{\kappa} \nabla\left(\frac{v_{g}}{3 n^{2}} E\right) .
$$

The group velocity depends on $n$; for a homogeneous medium we may cancel $n^{2}$, pull $v_{g}$ out from the gradient operator and thereby arrive at a familar expression, except with $c$ replaced by the group speed,

$$
\mathbf{F}=-\frac{v_{g}}{3 \kappa} \nabla E .
$$

Substituting the result into Eq.(4) yields the multifrequency diffusion equation,

$$
\partial_{t} E-\nabla \cdot \frac{v_{g}}{3 \kappa} \nabla E=\kappa v_{g}\left[4 \pi n^{2} \mathcal{B}_{\nu}(T) / v_{g}-E\right] .
$$


Hence, at equilibrium $E$ relaxes to $4 \pi n^{2} \mathcal{B}_{\nu}(T) / v_{g}$, as noted by Castor [3].

The RS of Eq.(7) denotes the power the radiation field exchanges with the medium. Its integral over all relevant frequencies equals the $K_{r, m}$ term of Eq.(1). Hence, the matter energy equation is,

$$
C \partial_{t} T=\nabla \cdot k_{m} \nabla T+S-\int_{\nu_{0}}^{\infty} d \nu \kappa v_{g}\left[4 \pi n^{2} \mathcal{B}_{\nu}(T) / v_{g}-E\right] .
$$

In Eq.(8) we introduce a frequency $\nu_{0}$ that designates the opaque interval $\left(0, \nu_{0}\right)$, Larsen et al, [11] and [12], where the opacity is so large, that $E$ equals the emission source, i.e.,

$$
\text { for } \nu \in\left(0, \nu_{0}\right), \quad E=4 \pi n^{2} \mathcal{B}_{\nu}(T) / v_{g} .
$$

It is instructive to examine how $E$ and $\mathbf{F}$ relate to a two term expansion of $I$ in terms of spherical harmonics,

$$
I(\boldsymbol{\Omega})=\frac{1}{4 \pi} I_{0}+\frac{3}{4 \pi} \boldsymbol{\Omega} \cdot \mathbf{I}_{1}
$$

where

$$
I_{0}=\int_{4 \pi} d \omega I \text { and } \mathbf{I}_{1}=\int_{4 \pi} d \omega \Omega I .
$$

It follows that $I_{0}=v_{g} E$ and $\mathbf{I}_{1}=\mathbf{F}$. Substituting for $\mathbf{F}$ from Eq.(6), yields

$$
I=\frac{v_{g}}{4 \pi}\left[E-3\left(\frac{1}{3 \kappa}\right) \boldsymbol{\Omega} \cdot \nabla E\right] .
$$

\section{$3 \quad$ Boundary conditions}

Equations (7) and (8) make up the system of interest. Both require appropriate boundary conditions. In next two Sections we first derive conditions for Eq.(7); then take up conditions for Eq.(8). The application of interest is a silica slab surrounded by air held at temperature $T_{a}$.

\subsection{Radiation boundary condition}

As discussed by Larsen et al, [11] and [12], at the boundary, $I\left(\doteq I_{b}\right)$ satisfies a condition for incoming directions $\Omega$,

$$
I=I_{b}(\boldsymbol{\Omega})=I_{b, p}(\boldsymbol{\Omega})+I_{b, r}(\boldsymbol{\Omega}) .
$$

The $I_{b, p}$ term on the RS is the "penetrating" radiation,

$$
I_{b, p}(\boldsymbol{\Omega})=[1-R(\mu)] \mathcal{B}_{\nu}\left(T_{a}\right),
$$


Note that $\mathcal{B}_{\nu}$ is evaluated at the air temperature $T_{a}$. The function $R(\mu)$ defines the reflectivity, Isard [9]. Its argument $\mu$ is the cosine of the angle that the incoming ray $\boldsymbol{\Omega}$ makes with $\hat{\mathbf{n}}$, the inward pointing normal: $\mu \doteq \hat{\mathbf{n}} \cdot \boldsymbol{\Omega}>0$. The reflectivity also varies with frequency via its dependence on $n$ and the nondimensional absorptivity index $k$, that defines the opacity: $\kappa=4 \pi k / \lambda$, where $\lambda$ is the wavelength. For normal-normal incidence,

$$
\left.R\right|_{\mu=0}=\frac{(n-1)^{2}+k^{2}}{(n+1)^{2}+k^{2}} .
$$

The second term $I_{b, r}$ on the RS of Eq.(11) stems from radiation propagating along outgoing directions $\boldsymbol{\Omega}^{\prime}$ that is reflected back into the domain,

$$
I_{b, r}(\boldsymbol{\Omega})=R(\mu) I\left(\boldsymbol{\Omega}^{\prime}\right), \quad \boldsymbol{\Omega}^{\prime}=\boldsymbol{\Omega}-2(\hat{\mathbf{n}} \cdot \boldsymbol{\Omega}) \hat{\mathbf{n}} .
$$

In a diffusion approximation, Eq.(11) can only be satisfied in an integral sense. We integrate over the hemisphere inside the glass, i.e., for $\hat{\mathbf{n}} \cdot \boldsymbol{\Omega}>0$. After integrating over the azimuthal angle, Eq.(11) becomes

$$
2 \pi \int_{0}^{1} d \mu \mu\left(I-I_{b}\right)=0 .
$$

The extra $\mu$ in the integrand is required since we are computing the flux normal to the interface, Zel'dovich and Raizer [24].

For the integral of $I$, we substitute the Eq.(9) expansion,

$$
2 \pi \int_{0}^{1} d \mu \mu I=\left(I_{0}+2 \mathbf{I}_{1} \cdot \hat{\mathbf{n}}\right) / 4
$$

where we used the properties,

$$
2 \pi \int_{0}^{1} d \mu \mu=\pi \text { and } \int_{2 \pi} d \omega \mu \boldsymbol{\Omega}=2 \pi / 3 .
$$

Substituting for the $I_{0}$ and $\mathbf{I}_{1}$ coefficients yields,

$$
2 \pi \int_{0}^{1} d \mu \mu I=\frac{v_{g}}{4}\left(E-\frac{2}{3 \kappa}(\hat{\mathbf{n}} \cdot \nabla E)\right) .
$$

For the integral over the penetrating part, we recall the argument of Isard [9]. Assume azimuthal symmetry and let $\theta$ define the angle that a ray, incoming from the air side, makes with the normal. Because of refraction, the transmitted ray $\boldsymbol{\Omega}$ makes a smaller angle $\phi$ with the inward normal $\hat{\mathbf{n}} ;$ see Figure 1.

The flux/area entering between angles $\theta$ and $\theta+d \theta$,

$$
[1-R(\theta)] 2 \pi I_{\text {air }} \cos (\theta) \sin (\theta) d \theta
$$




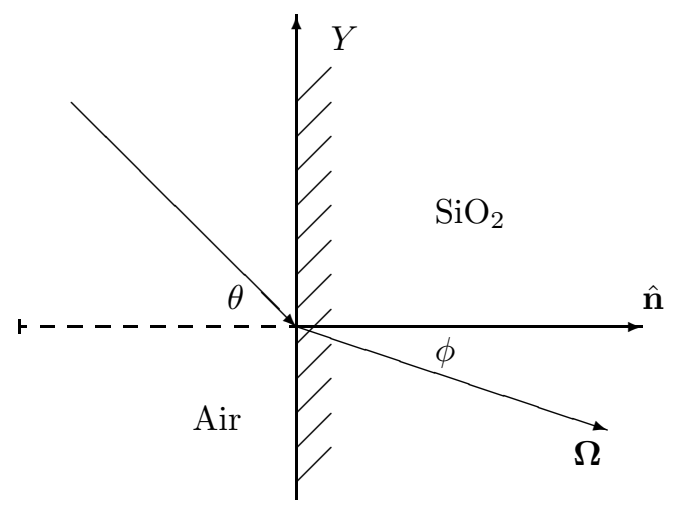

Figure 1: Schematic of penetrating ray.

is transmitted into a flux/area into angles between $\phi$ and $\phi+d \phi$,

$$
[1-R(\phi)] 2 \pi I_{\text {air }} \cos (\phi) \sin (\phi) d \phi .
$$

For refractive, lossy media, the angles $\theta$ and $\phi$ are related via a relationship significantly more complicated than a simple application of Snell's law. However, the air intensity $I_{\text {air }}=\mathcal{B}_{\nu}\left(T_{a}\right)$ is isotropic; hence, any off-normal incoming ray is balanced by a specularly symmetric counterpart, Isard [9]. Thus, it suffices to consider only normal incidence and in this case, Snell's law does link the two angles: $\sin (\theta)=n \sin (\phi)$. Hence,

$$
\cos (\theta) \sin (\theta) d \theta=n^{2} \cos (\phi) \sin (\phi) d \phi .
$$

This enables computing the integral over the penetrating part,

$$
\begin{aligned}
2 \pi \int_{0}^{1} d \mu \mu I_{b, p} & =2 \pi n^{2} \mathcal{B}_{\nu}\left(T_{a}\right) \int_{0}^{1} d \mu \mu[1-R(\mu)] \\
& =\pi\left(1-2 r_{1}\right) n^{2} \mathcal{B}_{\nu}\left(T_{a}\right)
\end{aligned}
$$

where $\mu=\cos (\phi)$ and

$$
r_{j} \doteq \int_{0}^{1} d \mu \mu^{j} R(\mu), \quad j=1,2, \ldots
$$

For the integral over the reflected intensity $I_{b, r}$, the outgoing radiation is also given by Eq.(9) except with $\boldsymbol{\Omega}$ replaced with $\boldsymbol{\Omega}^{\prime}$. Equation (10) implies,

$$
\begin{aligned}
\int_{2 \pi} d \omega \mu I_{b, r}(\boldsymbol{\Omega}) & =\int_{2 \pi} d \omega \mu R(\mu) I\left(\boldsymbol{\Omega}^{\prime}\right)=\frac{1}{2}\left[r_{1} I_{0}-3 r_{2}\left(\hat{\mathbf{n}} \cdot \mathbf{I}_{1}\right)\right] \\
& =\frac{v_{g}}{2}\left(r_{1} E+\frac{r_{2}}{\kappa}(\hat{\mathbf{n}} \cdot \nabla E)\right) .
\end{aligned}
$$


Substituting Eqs.(14), (15) and (16) into Eq.(13) yields,

$$
\frac{v_{g}}{4}\left[\left(1-2 r_{1}\right) E-\left(1+3 r_{2}\right)\left(\frac{2}{3 \kappa}\right)(\hat{\mathbf{n}} \cdot \nabla E)\right]=\left(1-2 r_{1}\right) \pi n^{2} \mathcal{B}_{\nu}\left(T_{a}\right) .
$$

The expression is put into familiar form by using the outward normal $-\hat{\mathbf{n}}$ :

$$
E+\left(\frac{1+3 r_{2}}{1-2 r_{1}}\right)\left(\frac{2}{3 \kappa}\right)(-\hat{\mathbf{n}} \cdot \nabla E)=4 \pi n^{2} \mathcal{B}_{\nu}\left(T_{a}\right) / v_{g}
$$

Since $-\nabla E$ is proportional to the radiation flux, the second term on the LS is proportional to the flux entering the domain. If $E$ is greater (less) than the RS, energy leaves (enters) the domain. At equilibrium, $E$ equals the RS and the flux shuts off, as expected.

\subsection{Temperature boundary condition}

We now consider the boundary condition for Eq.(8); the RS has three terms. The first is the divergence of the material energy flux. If we do not explicitly impose a boundary temperature, the exiting heat flux can be prescribed by a convection model, e.g.,

$$
-\hat{\mathbf{n}} \cdot\left(k_{m} \nabla T\right)=h_{m}\left(T_{a}-T\right),
$$

where $h_{m}$ is the (possibly nonlinear) convective coefficient and, as before, $\hat{\mathbf{n}}$ is the inward normal.

The integral term on the RS of Eq.(8) is the radiation-matter energy exchange rate. The interval $\left(0, \nu_{0}\right)$ denotes the opaque part of the spectrum where $E=4 \pi n^{2} \mathcal{B}_{\nu}(T) / v_{g}$. The integral extends only to $\nu_{0}$ since there is no energy exchange at lower frequencies. However, energy can either radiate out of, or into, the medium over the opaque interval.

The net radiation flux per frequency in the opaque interval is the difference between two one-way fluxes; one from air to glass; the other, glass to air. For the former, as in Sec. 3.1, the flux/area between angles $\theta$ and $\theta+d \theta$ is transmitted

into angles $\phi$ and $\phi+d \phi$. After integrating over the hemisphere, the flux entering the medium,

$$
F_{a \rightarrow g}=\pi\left(1-2 r_{1}\right) n^{2} \mathcal{B}_{\nu}\left(T_{a}\right) .
$$

In the opposite direction $g \rightarrow a$, the exiting flux/area exiting between angles $\phi$ and $\phi+d \phi$ is,

$$
[1-R(\mu)] 2 \pi I_{g} \cos (\phi) \sin (\phi) d \phi,
$$

where the glass intensity $I_{g}=n^{2} \mathcal{B}_{\nu}(T)$. Integrating over the hemisphere yields the flux leaving the medium,

$$
F_{g \rightarrow a}=2 \pi \int_{0}^{1} d \mu \mu I_{g}=\pi\left(1-2 r_{1}\right) n^{2} \mathcal{B}_{\nu}(T),
$$


The difference $F_{g \rightarrow a}-F_{a \rightarrow g}$ is the net outward flux. After integrating over $\nu$, substituting from Eqs.(19) and (18), we arrive at the boundary condition for the temperature,

$$
-\hat{\mathbf{n}} \cdot\left(k_{m} \nabla T\right)=h_{m}\left(T_{a}-T\right)+\pi\left(1-2 r_{1}\right) n_{0}^{2} \int_{0}^{\nu_{0}} d \nu\left[\mathcal{B}_{\nu}\left(T_{a}\right)-\mathcal{B}_{\nu}(T)\right],
$$

where $n_{0}$ denotes the refractive index for the opaque interval. (For $\mathrm{SiO}_{2}$, for low frequencies, $n_{0} \approx 2$, Kitamura [10].)

Equation (20) is nonlinear. For computational purposes, we linearize as follows. The system of interest is time-dependent; let a zero superscript denote values at the prior time cycle. The Planck function is linearized,

$$
\mathcal{B}_{\nu}(T)=\mathcal{B}_{\nu}^{0}+\mathcal{B}_{\nu}^{0, \prime}\left(T-T^{0}\right),
$$

where $\mathcal{B}_{\nu}^{0} \doteq \mathcal{B}_{\nu}\left(T^{0}\right)$ and $\left.\mathcal{B}_{\nu}^{0, \prime} \doteq\left(\partial \mathcal{B}_{\nu} / \partial T\right)\right|_{T=T^{0}}$. Inserting the linearization and rearranging terms yields a boundary condition of mixed type analogous to Eq.(17) for the radiation energy, viz.,

$$
\begin{aligned}
& \left(h_{m}+\pi\left(1-2 r_{1}\right) n_{0}^{2} \int_{0}^{\nu_{0}} d \nu \mathcal{B}_{\nu}^{0, \prime}\right) T-\hat{\mathbf{n}} \cdot\left(k_{m} \nabla T\right)= \\
& \quad h_{m} T_{a}+\pi\left(1-2 r_{1}\right) n_{0}^{2} \int_{0}^{\nu_{0}} d \nu\left[\mathcal{B}_{\nu}\left(T_{a}\right)-\left(\mathcal{B}_{\nu}^{0}-\mathcal{B}_{\nu}^{0, \prime} T^{0}\right)\right] .
\end{aligned}
$$

For stability, the parenthetical expression $\left(\mathcal{B}_{\nu}^{0}-\mathcal{B}_{\nu}^{0,}{ }^{\prime} T^{0}\right)$ on the RS is maxed with zero. If the convective coefficient $h_{m}$ varies with $T$, there is additional nonlinearity. In this case, it's simplest to let $h_{m}=h_{m}\left(T^{0}\right)$, i.e., to lag it temporally.

To summarize, the system of interest is,

$$
\begin{aligned}
\partial_{t} E & =\nabla \cdot \frac{v_{g}}{3 \kappa} \nabla E+\kappa v_{g}\left[4 \pi n^{2} \mathcal{B}_{\nu}(T) / v_{g}-E\right] \\
C \partial_{t} T & =\nabla \cdot k_{m} \nabla T+S-\int_{\nu_{0}}^{\infty} d \nu \kappa v_{g}\left[4 \pi n^{2} \mathcal{B}_{\nu}(T) / v_{g}-E\right] .
\end{aligned}
$$

The equations depend on position and time. Equation (22) also depends on frequency. The boundary conditions are of mixed type:

$$
\begin{gathered}
E+\left(\frac{1+3 r_{2}}{1-2 r_{1}}\right)\left(\frac{2}{3 \kappa}\right)(-\hat{\mathbf{n}} \cdot \nabla E)=4 \pi n^{2} \mathcal{B}_{\nu}\left(T_{a}\right) / v_{g} \\
-\hat{\mathbf{n}} \cdot\left(k_{m} \nabla T\right)=h_{m}\left(T_{a}-T\right)+\pi\left(1-2 r_{1}\right) n_{0}^{2} \int_{0}^{\nu_{0}} d \nu\left[\mathcal{B}_{\nu}\left(T_{a}\right)-\mathcal{B}_{\nu}(T)\right] .
\end{gathered}
$$

\section{Numerical scheme}

Equations(22)-(25) are similar to the multifrequency equations solved by conventional radiation-hydrodynamic codes. Thus, we consider well-known meth- 
ods of solution, the simplest of which is the "Partial Temperature" scheme of Lund and Wilson [14], [19].

We discretize the frequency domain into $G$ groups. Let $\left\{\nu_{i}\right\}_{i=0}^{G}$, where $\nu_{0}<$ $\nu_{1}<\nu_{2}<\ldots$, denote the group boundaries. The "opaque" interval is $\nu<\nu_{0}$. The last boundary $\nu_{G}$ denotes a frequency beyond which we don't expect to have much energy. In the applications, $\nu_{G}=10 \mathrm{eV}$ is large enough since we do not expect temperatures exceeding $3000(=0.26 \mathrm{eV})$.

We discretize Eqs.(22)-(23) using fully implicit temporal differencing. After multiplying by the timestep $\Delta t$ and integrating Eq.(22) over the $i$ th group, we obtain

$$
E_{i}-E_{i}^{0}=\nabla \cdot \frac{\Delta t v_{i}}{3 \kappa_{i}} \nabla E_{i}+\Delta t \kappa_{i}\left[B_{i}(T)-v_{i} E_{i}\right],
$$

where we define the group radiation energy,

$$
E_{i}=\int_{i} E \doteq \int_{\nu_{i}}^{\nu_{i+1}} d \nu E
$$

and $E_{i}^{0}$ is its analogue at the start of the time cycle. The $\kappa_{i}$ and $v_{i}$ coefficients are averages of $\kappa$ and $v_{g}$ over the $i$ th group. We discuss the averaging procedure in Section 5. The term

$$
B_{i}(T) \doteq 4 \pi n_{i}^{2} \int_{i} \mathcal{B}_{\nu}(T)
$$

where $n_{i}$ is an group-average of $n$. The units of $E_{i}$ differ from units $(E)$; the former is the radiation energy density $\left(\mathrm{erg} / \mathrm{cm}^{3}\right)$ of the $i$ th group. Similarly, units of $B_{i}(T)$ differ from units of $\mathcal{B}_{\nu}(T) ; B_{i}(T) / v_{i}$ and $E_{i}$ have the same units.

We now consider the temperature equation. Since the coupling term in Eq.(26) has units of energy density exchanged per group, the integral in Eq.(23) is replaced by a sum. After discretizing the time derivative and multiplying by $\Delta t$, we obtain,

$$
C\left(T-T^{0}\right)=\Delta t\left(\nabla \cdot k_{m} \nabla T+S-\sum_{i=1}^{G} \kappa_{i}\left[B_{i}(T)-v_{i} E_{i}\right]\right) .
$$

Discretization of the boundary conditions is straightforward. Equation (24) becomes,

$$
E_{i}+\left(\frac{1+3 r_{2, i}}{1-2 r_{1, i}}\right)\left(\frac{2}{3 \kappa_{i}}\right)\left(-\hat{\mathbf{n}} \cdot \nabla E_{i}\right)=B_{i}\left(T_{a}\right) / v_{i}
$$

where $r_{j, i}(j=1,2)$, is an average, similar to that for $n_{i}$, of the $j$ th moment of the reflectivity over the group. The temperature boundary condition stems from Eq.(21)

$$
\begin{aligned}
& {\left[h_{m}+\left(\frac{1-2 r_{1,0}}{4}\right) \frac{d B_{0}\left(T^{0}\right)}{d T}\right] T-\hat{\mathbf{n}} \cdot\left(k_{m} \nabla T\right)=} \\
& \quad h_{m} T_{a}+\left(\frac{1-2 r_{1,0}}{4}\right)\left[B_{0}\left(T_{a}\right)-\left(B_{0}\left(T^{0}\right)-\frac{d B_{0}\left(T^{0}\right)}{d T} T^{0}\right)\right],
\end{aligned}
$$


where, as in Eq.(27), $B_{0}(T) \doteq 4 \pi n_{0}^{2} \int_{0}^{\nu_{0}} \mathcal{B}_{\nu}(T) d \nu$.

To summarize, the multigroup system consists of Eqs.(26), (28), (29) and (30) where the group index $i=1, \ldots, G$.

\subsection{Partial Temperature scheme}

We now describe an application of the "Partial Temperature" for our multigroup system. We use operator splitting and first advance $T$ using only heat conduction and the source $S$ to produce an intermediate temperature $T^{(0)}$. For fully implicit differencing, we would solve,

$$
C\left(T^{(0)}-T^{0}\right)=\Delta t\left(\nabla \cdot k_{m} \nabla T^{(0)}+S\right) .
$$

Note that $T^{0}$ is the temperature at the start of the time cycle, while $T^{(0)}$ is an intermediate temperature. If matter-radiation coupling was neglected, $T^{(0)}$ is the temperature at the end of the time cycle.

Next, we rearrange group numbers into random order. We cycle through each group and solve a coupled system of two equations producing an updated radiation group energy $E_{i}$ and an intermediate (partial) temperature $T^{(i)}$. The two equations are Eq.(26) and

$$
C\left(T^{(i)}-T^{(i-1)}\right)=-\Delta t \kappa_{i}\left[B_{i}\left(T^{(i)}\right)-v_{i} E_{i}\right] .
$$

The process starts with the first randomly assigned group corresponding to $i=1$, and for this group, $T^{(i-1)}=T^{(0)}$, the result of advancing Eq. (31).

In Eqs.(26) and (32), the Planck function is linearized about $T^{(i-1)}$,

$$
B_{i}\left(T^{(i)}\right) \rightarrow B_{i, \ell}\left(T^{(i)}\right) \doteq B_{i}\left(T^{(i-1)}\right)+\frac{d B_{i}\left(T^{(i-1)}\right)}{d T}\left(T^{(i)}-T^{(i-1)}\right) .
$$

The linearization enables solving Eq.(32) for $\left(T^{(i)}-T^{(i-1)}\right)$ in terms of known quantities. After substituting into Eq.(26), we obtain

$$
E_{i}-E_{i}^{0}=\nabla \cdot \frac{\Delta t v_{i}}{3 \kappa_{i}} \nabla E_{i}+\Delta t \kappa_{i}^{\prime}\left[B_{i}\left(T^{(i-1)}\right)-v_{i} E_{i}\right]
$$

where

$$
\kappa_{i}^{\prime}=C \kappa_{i} /\left(C+\Delta t \kappa_{i} \frac{d B_{i}\left(T^{(i-1)}\right)}{d T}\right) .
$$

Equation (34) is a fully implicit discretization of a scalar reaction-diffusion equation with a known source. We rearrange it into a linear system for the unknown $E_{i}$ :

$$
\left(\left(1+\Delta t \kappa_{i}^{\prime} v_{i}\right) \mathbf{I}-\nabla \cdot \frac{\Delta t v_{i}}{3 \kappa_{i}} \nabla\right) E_{i}=E_{i}^{0}+\Delta t \kappa_{i}^{\prime} B_{i}\left(T^{(i-1)}\right),
$$


where $\mathbf{I}$ is the identity matrix. After solving for $E_{i}$, the updated temperature,

$$
T^{(i)}=T^{(i-1)}-\Delta t \kappa_{i}^{\prime}\left[B_{i}\left(T^{(i-1)}\right)-v_{i} E_{i}\right] / C .
$$

After cycling through all the groups, $T^{(G)}$ is the desired final temperature $T$. The scheme is conservative. The energy exchanged between matter and radiation is the sum of Eq.(32) for all $i$. The LS telescopes and we obtain,

$$
C\left(T-T^{(0)}\right)=-\Delta t \sum_{i=1}^{G} \kappa_{i}\left[B_{i, \ell}\left(T^{(i)}\right)-E_{i}\right],
$$

where $B_{i, \ell}\left(T^{(i)}\right)$ is given by the Eq.(33) linearization.

To summarize, we first obtain $T^{(0)}$ by solving Eq.(31). Next, we cycle through the groups in random order. For each $i, E_{i}$ is the solution of the linear system, Eq.(35). The $i$ th partial temperature is obtained from Eq.(36). The final temperature $T=T^{(G)}$.

\section{$5 \quad$ Material properties}

In this section we describe material properties relevant to our application and how the group averaged coefficients, e.g., $\kappa_{i}$ are defined. We obtain fundamental data by scanning the Kitamura et al results ([10], [22]) for the nondimensional indexes of refraction $n$ and absorption $k$. Figures 2 and 3 display $n$ and $k$, respectively, over our frequency range of interest: $10^{-3}<\nu<10$. (Henceforth, we measure $\nu$ in $\mathrm{eV} ; 1 \mathrm{eV} \approx 2.4 \cdot 10^{14} \mathrm{~Hz}$.) The figures also display three group-averaged sets.

We arrive at the $\left(10^{-3}, 10\right) \mathrm{eV}$ range using an energy argument. We expect temperatures in the range $(300,3000)^{\circ} \mathrm{K}$, i.e., $(0.0259,0.259) \mathrm{eV}$. The integral of the normalized Planckian $b(y)=y^{3} /\left(e^{y}-1\right)$, where $y \doteq h_{P} \nu / k_{K} T$, for all $y$, is $\pi^{4} / 15=6.49$. If we choose a sufficiently small $\nu_{0}$ and a large enough $\nu_{G}$ so that integrals over ranges $\left(0, y_{0}\right)$ and $\left(y_{G}, \infty\right)$ are negligibly small in comparison with 6.49 , radiation energy emission over those ranges is also negligible. The choices $y_{0}=0.1$ and $y_{G}=20$ give integral ratios of $5 \cdot 10^{-5}$ and $3 \cdot 10^{-6}$, respectively. Thus, by choosing $\nu_{0}<0.1 \times 0.0259 \mathrm{eV}$ and $\nu_{G}>20 \times 0.259 \mathrm{eV}$, we obtain a sufficiently broad spectrum for the temperatures we plan to encounter.

Group averages are obtained as follows. Data scanned from [22] are interpolated onto the same array of frequencies creating a set of $(\nu, n, k)$ triplets, of which approximately 1000 lie within the interval $10^{-3}<\nu<10$. The expression $\kappa=4 \pi k / \lambda$, where $\lambda$ is the wavelength, generates an opacity from each $(\nu, k)$ pair. Next, we construct six sets of logarithmically spaced group boundaries $\left\{\nu_{i}\right\}_{i=0}^{G}$, successively refining each set by a factor of two. The coarsest set has four intervals (one per eV decade); the finest, 128. For each set, the lowest group boundary $\nu_{0}=10^{-3} \mathrm{eV}$ and the highest $\nu_{G}=10 \mathrm{eV}$. We then form group 


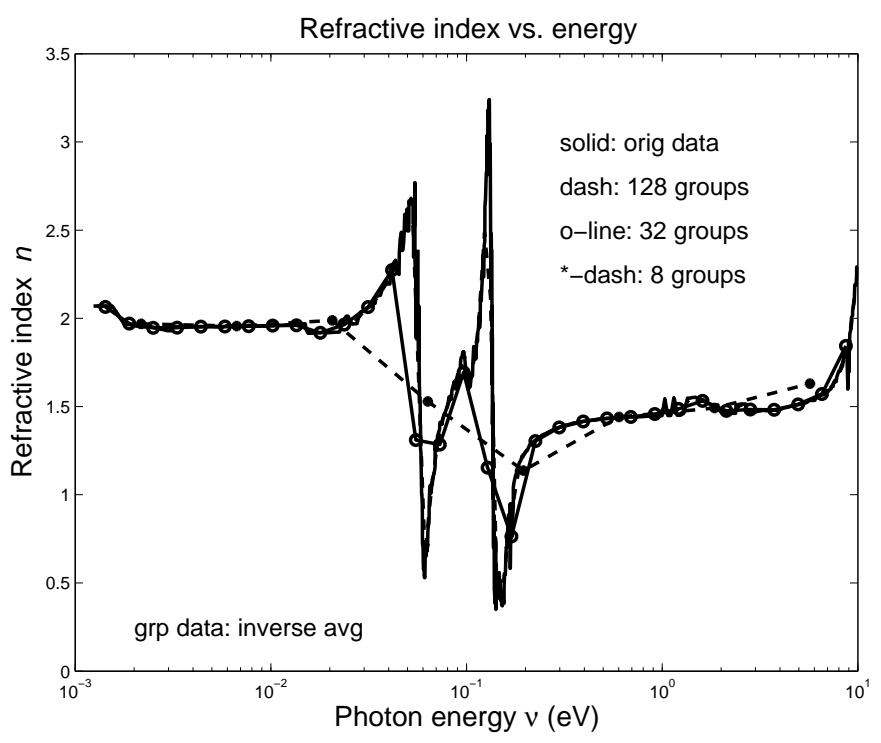

Figure 2: Refractive index $n$ vs. $\nu$.

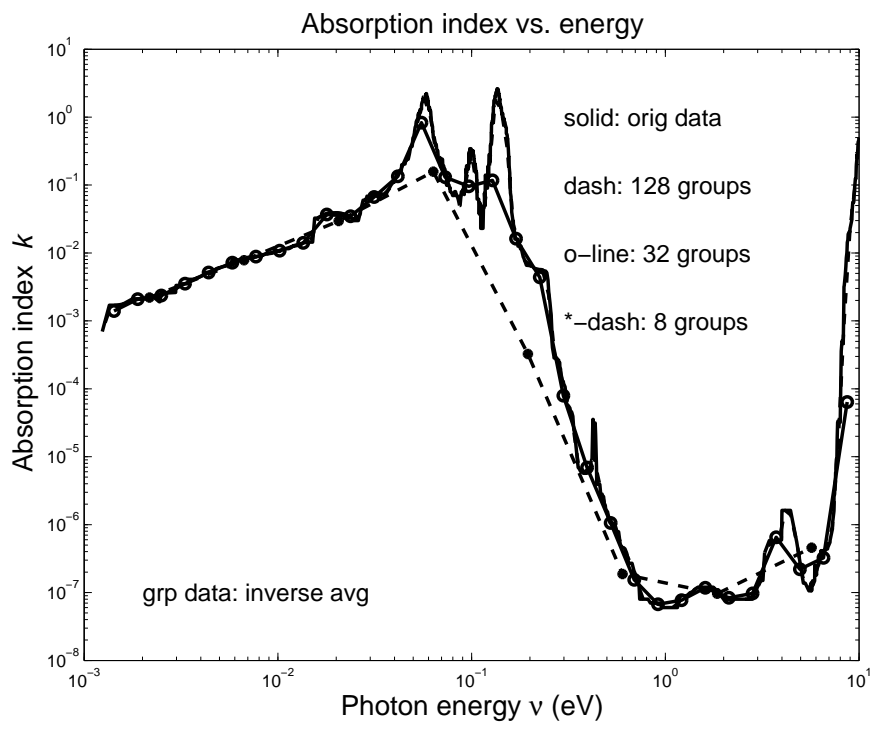

Figure 3: Absorption index $k$ vs. $\nu$. 


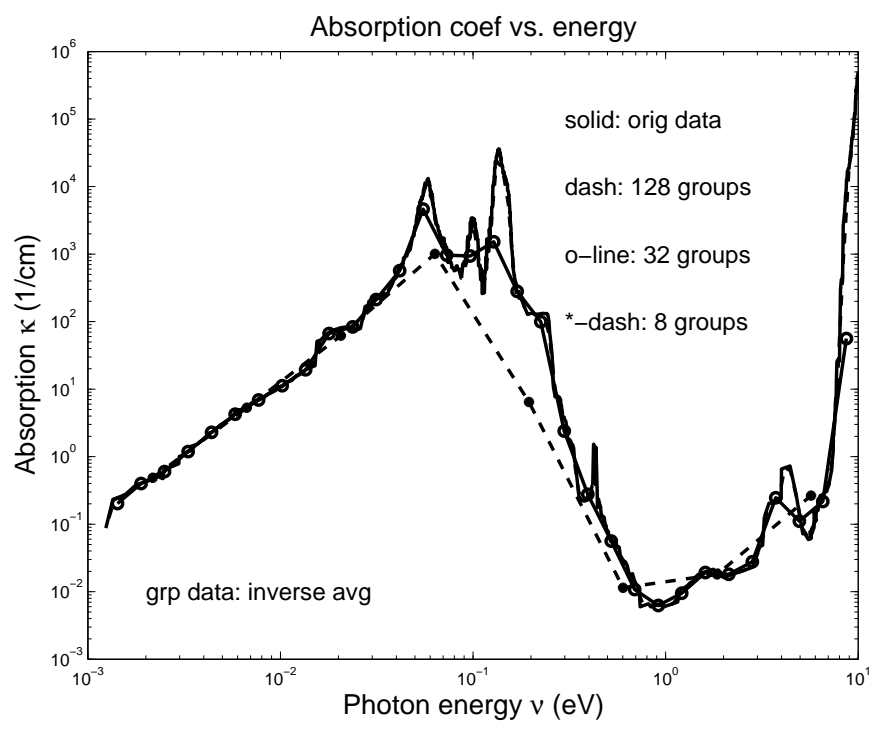

Figure 4: Opacity $\kappa$ vs. $\nu$.

averages of $\kappa$ and $n$ over each interval. We average $\kappa$ (instead of $k$ ) since the opacity is the relevant coefficient. We use inverse averaging,

$$
\left(1 / \kappa_{i}\right) \doteq\left(\int_{\nu_{i-1}}^{\nu_{i}} d \nu / \kappa\right) /\left(\nu_{i}-\nu_{i-1}\right),
$$

and similarly for $n_{i}$. Figure 4 displays $\kappa$ and three group-averaged analogues. The figure shows that 128 groups overlay nicely with the data, while hinting that 32 groups may be insufficient to obtain accurate results.

We inverse average the opacity for the following reasons. (a) Inverse averaging $\kappa$ is equivalent to Rosseland averaging, if $\partial \mathcal{B}_{\nu} / \partial T$ was constant. (b) Rosseland averaging is preferred for multigroup radiation transport [18]. (c) We use simple averaging since we only have data at room temperature. If the discretization is fine enough, how to average is immaterial while for a coarse discretization, it seems unreasonable to favor opacities near the peak of $\partial \mathcal{B}_{\nu} / \partial T$ evaluated at only $T=300^{\circ} \mathrm{K}$. Nevertheless, $\kappa$ can vary significantly with $T$. For example, McLachlan and Meyer [15] report a nearly linear increase of $k$ with $T$ for a few wavelengths. In silica, for $\lambda=10.59 \mu \mathrm{m}(0.117 \mathrm{eV})$, the mean free path $\kappa^{-1}$ decreases nearly tenfold from $40.7 \mu \mathrm{m}$ at $25{ }^{\circ} \mathrm{C}$ to $4.2 \mu \mathrm{m}$ at $1800{ }^{\circ} \mathrm{C}$. Hence, since in this paper we assume a homogeneous medium, our results can only give a qualitative effect of radiation transport. For quantitative details, we require data, or a model, with a temperature dependence for $k$ and $n$ for all relevant $\nu$, and, of course, use the more complicated radiation equation that includes non-homogeneous effects [17]. 


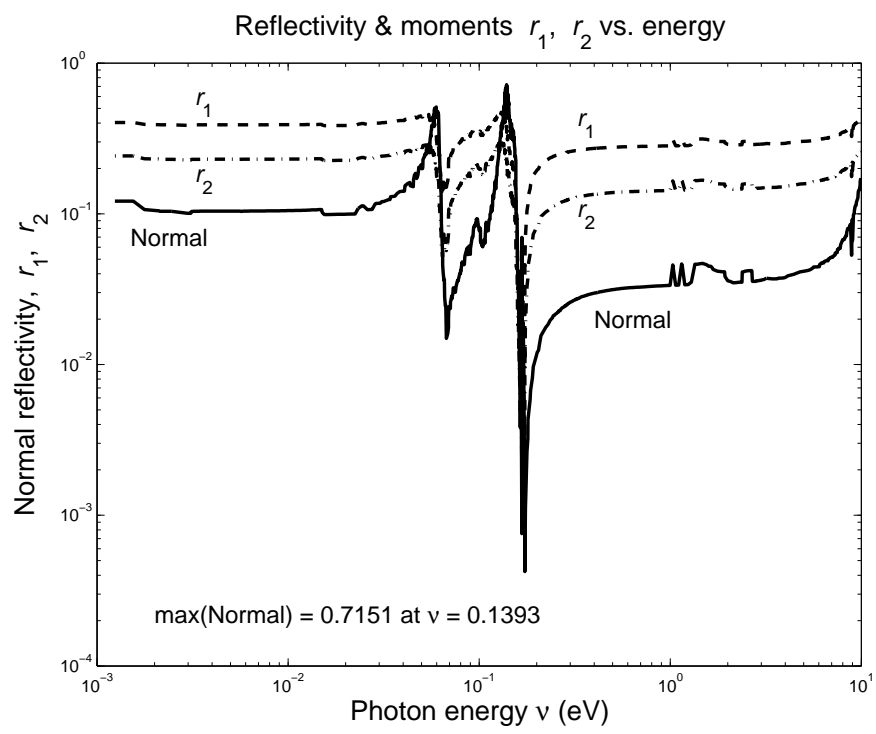

Figure 5: Normal incidence reflectivity $R \mid \mu=0$ and moments $r_{1}$ and $r_{2}$ vs. photon energy $\nu$.

Besides the group-averaged $\kappa_{i}$, we also need $v_{i}$, a group-average of the speed $v_{g}$ defined in Eq.(3). Since our basic (scanned) data is noisy, we ignore the $d n / d \nu$ derivative and for the simulations, set $v_{g}=v_{p}=c / n$. The group averaged $n_{i}$ then defines $v_{i}=c / n_{i}$. For intervals where $n_{i}<1$, we set $v_{i}=c$.

We now discuss the reflectivity function $R$ of Eq.(12) and the moments $r_{j}$ that arise in the boundary conditions. Figure 5 displays $R$ for normal incidence, i.e., for $\mu=0$. Note the large variation near $\nu=0.15 \mathrm{eV}$. Over a small frequency interval, $R$ attains a maximum of 0.7 and a minimum of $5 \cdot 10^{-4}$, which implies that in a narrow frequency range, for normal incidence, $\mathrm{SiO}_{2}$ either reflects over $70 \%$ of the incident light or less that $0.1 \%$.

For off-normal incidence, $R$ increases. As discussed by Isard [9], a ray incident at angle $\theta \in(0, \pi / 2)$ is usually ${ }^{4}$ transmitted into a smaller angle $\phi$. For normal incidence, $\theta=0$ transmits into $\phi=0$; for grazing incidence, $\theta=\pi / 2$ transmits into the critical angle $\phi_{\text {crit }}$. For $\phi>\phi_{\text {crit }}, R=1$ (total reflection). Since $\phi_{\text {crit }}$ depends on $n$ and $k$, Isard [9], it varies with $\nu$. For $\nu \approx 0.13$, $\phi_{\text {crit }} \approx 18^{\circ}$, while for $\nu \approx 0.17, \phi_{\text {crit }} \approx 89^{\circ}$.

Figure 5 also displays the $r_{1}$ and $r_{2}$ moments. As expected, $r_{1}>r_{2}$ and $r_{1}<$ 0.5 ; the latter relation is necessary since the boundary conditions of Eqs.(17) and (20) contain the term $\left(1-2 r_{1}\right)$, which must be positive.

\footnotetext{
${ }^{4}$ Except in regions of anomalous absorption where $n=k$ or $n<1$; see Isard's relation for the two angles $\theta$ and $\phi$.
} 
Lastly, we consider the opaque frequency interval $\left(0, \nu_{0}\right)$, introduced by Larsen [11] and [12]. The interval leads to the integral in the boundary condition Eq.(25) which stems from assuming that the opacity in the opaque interval is so large that radiation energy is in equilibrium with the matter emission source, i.e., $E=4 \pi n^{2} \mathcal{B}_{\nu}(T) / v_{g}$. Unfortunately, the data belies the assumption; in Figure $4, \kappa$ increases with $\nu$ in the range $\left(10^{-3}, 0.03\right) \mathrm{eV}$. Since we do not have data for smaller frequencies, we are unable to confirm the equilibrium argument. Nevertheless, by including an opaque interval in the boundary condition, we at least allow energy to radiate out. The error by making the equilibrium assumption should be small since, as stated above, there is little energy in the opaque interval.

\section{Simulations}

To simplify and enable future comparisons, we use materials with constant, albeit realistic, properties, viz., the coefficients of Bouchut et al [2] for the heat capacity $C$ and thermal conductivity $k_{m}$ :

$$
C=2.201 \cdot 10^{7}\left(\mathrm{erg} / \mathrm{cc}^{\circ} \mathrm{K}\right) \text { and } k_{m}=2.201 \cdot 10^{5}\left(\mathrm{erg} / \mathrm{cm} \mathrm{sec}^{\circ} \mathrm{K}\right) .
$$

For simulations with convective heat loss, we use a constant convectivity,

$$
h_{m}=1.243 \cdot 10^{4}\left(\mathrm{erg} / \mathrm{cm}^{2} \mathrm{sec}^{\circ} \mathrm{K}\right) .
$$

The constant $h_{m}$ was set by requiring that the loss term $h_{m}\left(T_{a}-T\right)$ in Eq.(20) yield the same flux as the nonlinear model of Stölken [21] when $T=1000^{\circ} \mathrm{K}$. The model stems from comparing to expressions for the Nusselt number, as discussed by Churchill and Chu [4]. The value conforms to the $10^{4}-10^{5}$ range (in CGS) found on the web [6].

We present results of both global, e.g., total energy in the domain, and local nature, such as $T$ and the radiation temperature $T_{r}$, where the latter is defined as follows. Given an energy density distribution per group $\left\{E_{i}\right\}_{i=1}^{G}$,

$$
T_{r} \doteq\left(\frac{1}{4 \sigma} \sum_{i=1}^{G} v_{i} E_{i} / n_{i}^{2}\right)^{(1 / 4)}
$$

where $\sigma$ is the Stefan-Boltzmann constant. The temperature $T_{r}$ can gauge of how close the radiation field is in equilibrium with the matter. The $T_{r}$ definition stems from Eqs.(26) and (27); $T_{r}$ is the temperature required for the total emission $\sum_{i=1}^{G} B_{i}\left(T_{r}\right) / v_{i}$ to equal the total radiation energy density $\sum_{i=1}^{G} E_{i}$.

In the next Section we present $1 \mathrm{D}$ results that simulate the cooling of a silica slab, initially at $T=2500^{\circ} \mathrm{K}$. In Section 6.2 , we simulate a disk of silica irradiated by a $\mathrm{CO}_{2}$ laser. 


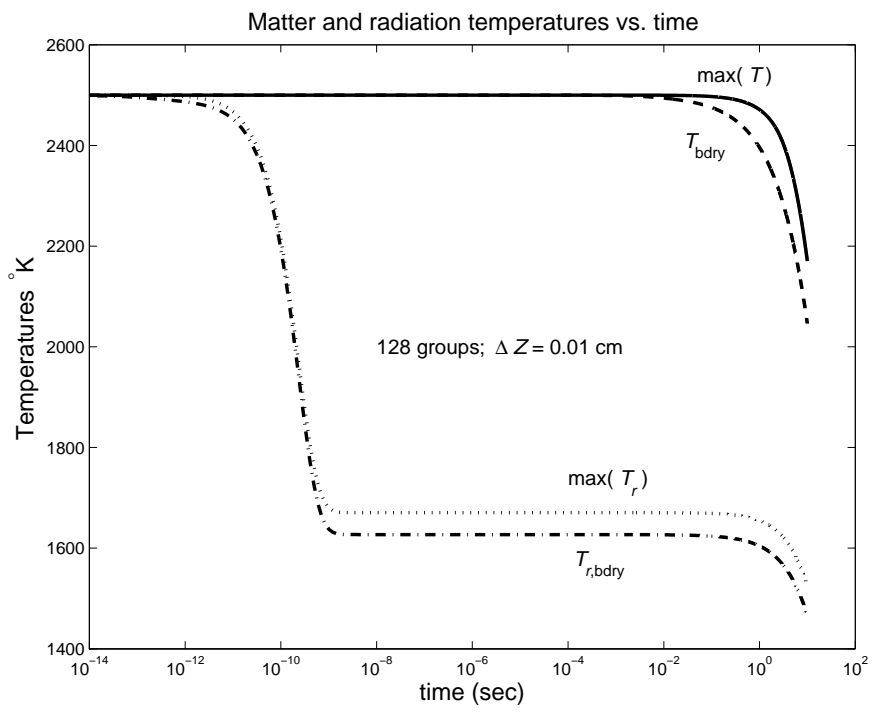

Figure 6: Silica cooling problem; temperatures $T$ and $T_{r}$ vs. time.

\subsection{Cooling of silica slab}

Our computer code is fundamentally $3 \mathrm{D}$, which, here, we run in axisymmetric $(R, Z)$ mode. The numerical domain is $R<10^{-3} \mathrm{~cm}$ and $0<Z<0.5 \mathrm{~cm}$. We impose symmetry at $Z=0.5$ and at $R=10^{-3}$; hence, model cooling of an infinitely long, $1 \mathrm{~cm}$ thick sheet of silica. Initially, radiation and matter are in equilibrium, $T=T_{r}=2500{ }^{\circ} \mathrm{K}$. At $Z=0$, the sheet is cooled strictly by radiative losses, i.e., in Eq.(25), the convection coefficient $h_{m}=0$. The exterior temperature $T_{a}$, used in Eqs.(24) and (25), is fixed at $298.15^{\circ} \mathrm{K}$. We simulate until $t=10 \mathrm{sec}$.

In the absence of radiative losses, the temperatures will not change. However, with radiation the sheet eventually cools to $T=298.15$. An interesting dynamic ensues. Figure 6 displays $\max (T)$ and $\max \left(T_{r}\right)$, as well as their values at the boundary. The figure shows the radiation field quickly reacts to the boundary condition; the boundary $T_{r}$ starts dropping at $t \approx 10^{-13} \mathrm{sec}$. As $T_{r \text {, bdry }}$ cools, so does the entire radiation field. During the decrease, the radiation field is nearly constant in space, as evidenced by the overlapping curves for $10^{-11}<t<10^{-9}$. Afterwards, in the range, $10^{-9}<t<0.01$, we have a near steady state, in which radiation loses energy (heat), but the matter temperature, because of the higher heat capacity, appears to be unchanged. However, for $t>0.01$, the slab begins to cool, first at the boundary, then throughout.

The dynamic response is summarized in Table 1, which displays the total initial matter and radiation energies $\mathcal{E}_{0}$ and $\mathcal{E}_{0, r}$, respectively, their final values $\mathcal{E}$ 


\begin{tabular}{ccccc}
\hline $\mathcal{E}_{0}$ & $\mathcal{E}$ & $\mathcal{E}_{0, r}$ & $\mathcal{E}_{r}$ & $\mathcal{E}_{f}$ \\
\hline $1.376 \cdot 10^{4}$ & $1.170 \cdot 10^{4}$ & $2.264 \cdot 10^{-7}$ & $2.823 \cdot 10^{-8}$ & $2.052 \cdot 10^{3}$ \\
\hline
\end{tabular}

Table 1: Slab cooling problem. Total initial, and final $(t=10 \mathrm{sec})$ matter and radiation energies $\mathcal{E}_{0}, \mathcal{E}, \mathcal{E}_{0, r}, \mathcal{E}_{r}$, and energy loss $\mathcal{E}_{f}$; (erg/radian) for domain: $0<R<10^{-3}, 0<Z<0.5 \mathrm{~cm}$; symmetry imposed at $Z=0.5$. To obtain values for disk with radius $R=10^{-3}$, multiply by $4 \pi$.

and $\mathcal{E}_{r}$, and the total fluence out the boundary $\mathcal{E}_{f}$. The table shows that while there is a $10^{11-12}$ disparity beween the energies in the matter and radiation fields, the latter acts as an efficient conduit for heat loss. By $t=10$, nearly $15 \%$ of the initial matter energy coupled to the radiation field and radiated away.

The results displayed in Figure (6) are confirmed by the following analysis. As stated by Gardon [7]: "In transparent materials, ... emission and absorption of radiation are bulk, rather than surface phenomena." Hence, for this problem, the first thing to happen is for the photons initially in the slab to exit. This happens quickly. The photon speed is approximately $c / \bar{n}$, where $\bar{n}$ is a frequency-averaged $n$. Figure 2 shows that $\bar{n} \doteq 1.7$ is a reasonable choice. Photons stream in all directions; some travel straight toward the surface while others in oblique angles. In any case, since the slab width $L=1 \mathrm{~cm}$, we expect the initial photons to exit at time $t_{0}=L /(c / \bar{n}) \approx 6 \cdot 10^{-11} \mathrm{sec}$. The estimate is in agreement with Fig.(6). By $t=10^{-9}$ sec, the initial photons are gone and we have a quasi steady state. This state lasts until the matter emits a sufficient amount of energy for $T$ to decrease. The time $t_{1}$ for this to occur follows by recalling Eq.(8) and ignoring everything but emission, i.e.,

$$
C \partial_{t} T=-4 \pi \int_{\nu_{0}}^{\infty} d \nu \kappa n^{2} \mathcal{B}_{\nu}(T)
$$

Defining a frequency averaged opacity $\bar{\kappa}$ leads to $\partial_{t} T=-\alpha T^{4}$, where $\alpha=$ $4 \sigma \bar{\kappa} \bar{n}^{2} / C$ and $\sigma$ is the Stefan-Boltzmann constant. The solution is,

$$
T / T_{0}=\left(1+3 \alpha T_{0}^{3} t\right)^{-1 / 3},
$$

where $T_{0}=2500$ is the initial temperature. Setting the LS to 0.99 and solving for $t$ gives the time $t_{1}$, when the temperature drops by 1\%. Using Eq.(37) to define $C$ yields $t_{1}=0.063 / \bar{\kappa} \bar{n}^{2}$. To get a numerical value, we need $\bar{\kappa}$ and $\bar{n}$. For the latter, we again use $\bar{n}=1.7$. For the former, we recall Fig.4. Despite the fact that $\kappa \approx 3 \cdot 10^{4}$ at $\nu \approx 0.13$ and $\kappa=\mathcal{O}\left(10^{5}\right)$ near $\nu=10, \kappa<1$ over the significantly large interval $0.3<\nu<7$. Hence, a reasonable estimate is $\bar{\kappa}=1$. Substituting, yields $t_{1}=0.022$, which agrees nicely with the time in Fig. 6 when $T$ begins to decrease. Lastly, we analyze the time difference between the initial temperature decrease of $\max (T)$ and $T_{\mathrm{bdry}}$, i.e., the delay between the decrease in the middle of the slab and at $Z=0$. If the decrease is due to heat conduction, i.e., the time for a diffusion wave, which starts at $Z=0$ to reach the midplane, 


\begin{tabular}{cccccccc}
\hline$G$ & $\mathcal{E} \cdot 10^{4}$ & $\mathcal{E}_{r} \cdot 10^{-8}$ & $\mathcal{E}_{f} \cdot 10^{3}$ & $\max (T)$ & $T_{\text {bdry }}$ & $\max \left(T_{r}\right)$ & $T_{r, \text { bdry }}$ \\
\hline 4 & 1.277 & 1.562 & 0.9907 & 2324.05 & 2311.36 & 1241.25 & 1237.75 \\
8 & 1.221 & 1.492 & 1.548 & 2274.42 & 2137.89 & 1450.87 & 1318.82 \\
16 & 1.184 & 2.236 & 1.919 & 2195.84 & 2077.93 & 1498.45 & 1428.63 \\
32 & 1.172 & 2.446 & 2.038 & 2170.95 & 2050.61 & 1516.62 & 1456.68 \\
64 & 1.172 & 2.688 & 2.033 & 2173.06 & 2051.15 & 1523.54 & 1465.49 \\
128 & 1.170 & 2.823 & 2.052 & 2170.27 & 2046.03 & 1525.15 & 1467.01 \\
\hline
\end{tabular}

Table 2: Slab cooling problem; group convergence study, $t=10 \mathrm{sec}$. Final matter and radiation energies $\mathcal{E}, \mathcal{E}_{r}$, and energy loss $\mathcal{E}_{f}$ (erg/radian). Final matter, radiation maximum and boundary temperatures $\left({ }^{\circ} \mathrm{K}\right)$.

\begin{tabular}{cccccccc}
\hline$N$ & $\mathcal{E} \cdot 10^{4}$ & $\mathcal{E}_{r} \cdot 10^{-8}$ & $\mathcal{E}_{f} \cdot 10^{3}$ & $\max (T)$ & $T_{\text {bdry }}$ & $\max \left(T_{r}\right)$ & $T_{r, \text { bdry }}$ \\
\hline 50 & 1.172 & 2.446 & 2.038 & 2170.95 & 2050.61 & 1516.62 & 1456.68 \\
100 & 1.174 & 2.459 & 2.013 & 2173.92 & 2058.49 & 1518.29 & 1452.42 \\
200 & 1.176 & 2.468 & 1.995 & 2176.00 & 2063.76 & 1519.45 & 1449.43 \\
400 & 1.177 & 2.475 & 1.983 & 2177.49 & 2067.31 & 1520.29 & 1447.47 \\
\hline
\end{tabular}

Table 3: Slab cooling; mesh convergence study. Same units as in Table 2.

the relevant equation is $\partial_{t} T=k^{\prime} \partial_{Z Z} T$, where $k^{\prime}=k_{m} / C=0.01$ is the ratio of the conductivity and specific heat. Recalling the fundamental solution of the heat equation, $L^{2} / 4 k^{\prime} t=\mathcal{O}(1)$, where $L$ is a characteristic length. Substituting for $k^{\prime}$ and letting $L=0.5 \mathrm{~cm}$, yields a characteristic time for the wave to reach the midplane, $t_{c}=6.25 \mathrm{sec}$, which is reasonable agreement with Fig.6.

We now discuss convergence; first, with group number, then, with mesh size. In all cases, we start with a small time step, $\Delta t=10^{-15}$ and let it increase appropriately to a maximum $\Delta t_{\max }=10^{-3}$, which ensures reasonable accuracy for the final decrease of temperature $(t>0.01)$. For convergence with number of groups $G$, we fix the mesh size at $\Delta Z=0.01$. Results appear in Table 2. The table shows that if one is interested in ca. $1 \%$ accuracy in $\max (T), 16$ groups is insufficient.

We next present convergence with mesh size. For all cases, we use 32 groups. We start with the same mesh width used for the results in Table 2, i.e., $N=50$ cells $(\Delta Z=0.01)$ and increase $N$ as shown in Table 3. Results show that if one is interested in only global quantities, e.g., $\mathcal{E}$, or in bulk properties, e.g., $\max (T)$, the coarsest mesh, $\Delta Z=0.01$, suffices. However, near the boundary, even 400 cells, $\Delta Z=1.25 \cdot 10^{-3}$, is not enough to attain a temperature accurate to three figures.

Lastly, in Table 4, we present results comparing the effects due to refraction, group velocities, reflectivity moments, and convection. Four results are displayed; all cases use $G=32$ groups and $\Delta Z=0.01$. Case "X" represents 


\begin{tabular}{cccccccc}
\hline Case & $\mathcal{E} \cdot 10^{4}$ & $\mathcal{E}_{r} \cdot 10^{-8}$ & $\mathcal{E}_{f} \cdot 10^{3}$ & $\max (T)$ & $T_{\text {bdry }}$ & $\max \left(T_{r}\right)$ & $T_{r, \text { bdry }}$ \\
\hline $\mathrm{X}$ & 1.188 & 0.731 & 1.878 & 2215.51 & 2051.94 & 1427.33 & 1296.72 \\
$\mathrm{R}$ & 1.080 & 1.422 & 2.960 & 2031.19 & 1832.46 & 1361.95 & 1226.73 \\
$\mathrm{~A}$ & 1.172 & 2.446 & 2.038 & 2170.95 & 2050.61 & 1516.62 & 1456.68 \\
$\mathrm{C}$ & 1.162 & 2.399 & 2.013 & 2160.02 & 2020.60 & 1510.45 & 1448.27 \\
\hline
\end{tabular}

Table 4: Slab cooling; effects of refraction, group speeds and reflectivity, see text. Same units as in Table 2.

conventional multigroup diffusion, i.e., we set $n_{i}=1, v_{i}=c$, and $r_{1}=r_{2}=0$. Case "R" is a simulation in which we use the $n_{i}$ and $v_{i}$ coefficients described above, but set the moments $r_{1}$ and $r_{2}$ to zero. Case "A" restates results of Table 2, line 4; refraction, group velocity, and reflectivity are used. Case "C" is similar to "A," except we include convection losses with $h_{m}$ as shown in Eq.(38). Comparing cases "A" and "R" illustrates the effect of reflectivity. Without it, the ratio of energy leaked $2.96 / 2.04$ is $45 \%$ larger, which yields a lower $\max (T)$ (2031 vs. $2171^{\circ} \mathrm{K}$ ). Cases "A" and "X" pertain to different coefficients. One striking difference is in the final energies $\mathcal{E}_{r}(0.7$ vs. 2.4) despite the similarity in $\max \left(T_{r}\right)$. The reason is that in case "A," the radiation is a modified Planckian; it contains the square of the refractive index. Since $n$ is approximately 1.52.0 , the square, $2.2-4$, is enough to raise $\mathcal{E}_{r}$ for case "X" to that of case "A." Comparing cases "A" and "C" show the effects of convection; with it the final energies and temperatures are lower since convection provides another heat loss mechanism.

\subsection{Laser annealing}

In contrast to the 1D slab-cooling simulation presented in Section 6.1, where the initial temperature gradient is zero, here we consider simulations with strong temperature gradients in both $1 \mathrm{D}$ and $2 \mathrm{D}$ axisymmetric geometries. We model heating a silica slab, initially at room temperature, with a $10.6 \mu \mathrm{m}$ wavelength $\mathrm{CO}_{2}$ laser. We use the same laser absorption parameters as Bouchut et al [2]. Laser energy is deposited into the silica as an explicit, position dependent, source, the term $S$ in Eq.(1), using a ray-tracing scheme that accounts for the reduction in beam flux due to absorption. Thermally insulated boundary conditions are applied; hence, energy can escape only via radiation. In both 1D and 2D geometries, we compare results from two simulations; one uses only heat conduction (HC) while the other couples $\mathrm{HC}$ with radiation diffusion. Parameters are chosen so that both $1 \mathrm{D}$ and $2 \mathrm{D}$ simulations give approximately the same peak temperature. Cases with radiation transport use 32 frequency groups.

The 1D simulations use a mesh similar to that described in Section 6.1. A detailed mesh and time step size convergence study show that $100 \mathrm{~nm}$ mesh resolution near the surface and time steps limiting temperature changes to $1^{\circ} \mathrm{K} /$ step 


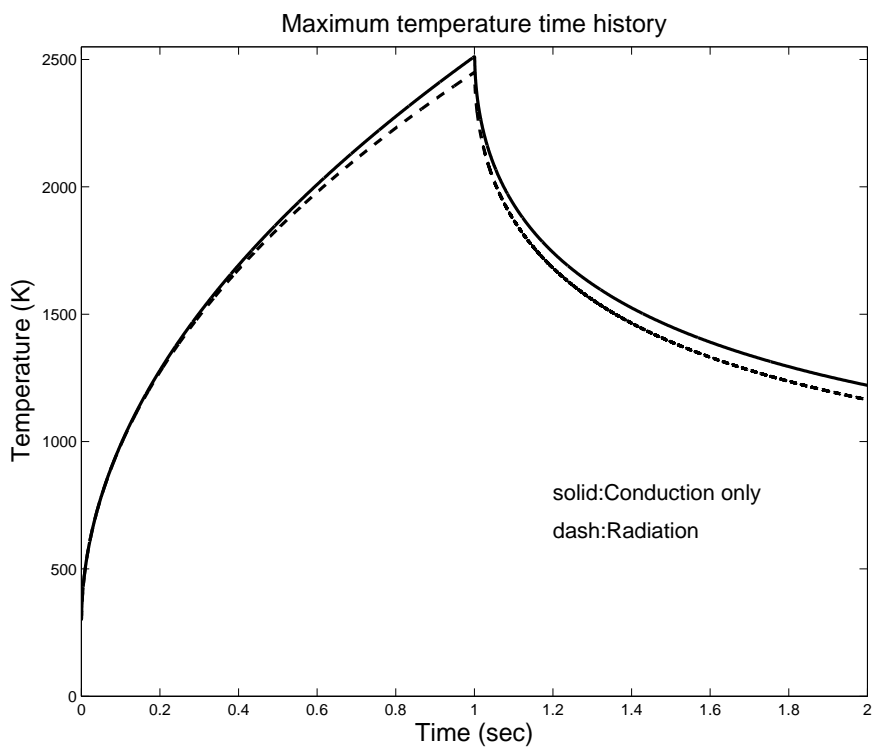

Figure 7: Maximum temperature vs time for 1D simulation with a 1s exposure and $4.34 \mathrm{~W} / \mathrm{mm}^{2}$ applied laser flux

suffice to capture variations in maximum temperature. Figure 7 displays time histories of $\max (T)$ of a simulation using only thermal conduction and one using both conduction and radiation. Radiation transport reduces the peak temperature by approximately $70^{\circ} \mathrm{K}$. The $70^{\circ} \mathrm{K}$ difference represents a lower bound for the actual effect because of the following argument. We use room temperature absorption data and it is known that generally, over the IR spectrum, the absorption coefficient $\kappa$ increases with temperature. (For $\lambda=10.6 \mu \mathrm{m}, \kappa$ changes by an order of magnitude over the indicated temperature range [15].) As shown in Eq.(2), the rate of energy transferred from matter to radiation is proportional to $\kappa$. Thus, a larger $\kappa$ results in a proportionally larger source of energy into the radiation field thereby enhancing its effect.

For the 2D axially symmetric simulations, energy is deposited using a laser with a Gaussian radial profile. The simulated domain is $8 \mathrm{~mm}$ in diameter and $4 \mathrm{~mm}$ thick. Mesh resolution near the absorbing surface is $100 \mathrm{~nm}$ and is gradually coarsened away from the laser spot yielding approximately 36000 computational nodes. The ray-tracing laser energy deposition scheme described above models the Gaussian radial beam profile. The beam's total power is $10 \mathrm{~W}$ and has a (1/e)-diameter of $1 \mathrm{~mm}$. Figure 8 displays axial and surface temperature profiles of two simulations; as for $1 \mathrm{D}$, one using only conduction and one coupling conduction with radiation. The modest reduction in temperature $\left(\approx 50^{\circ} \mathrm{K}\right)$ due to radiation represents a lower bound for the actual effect for 


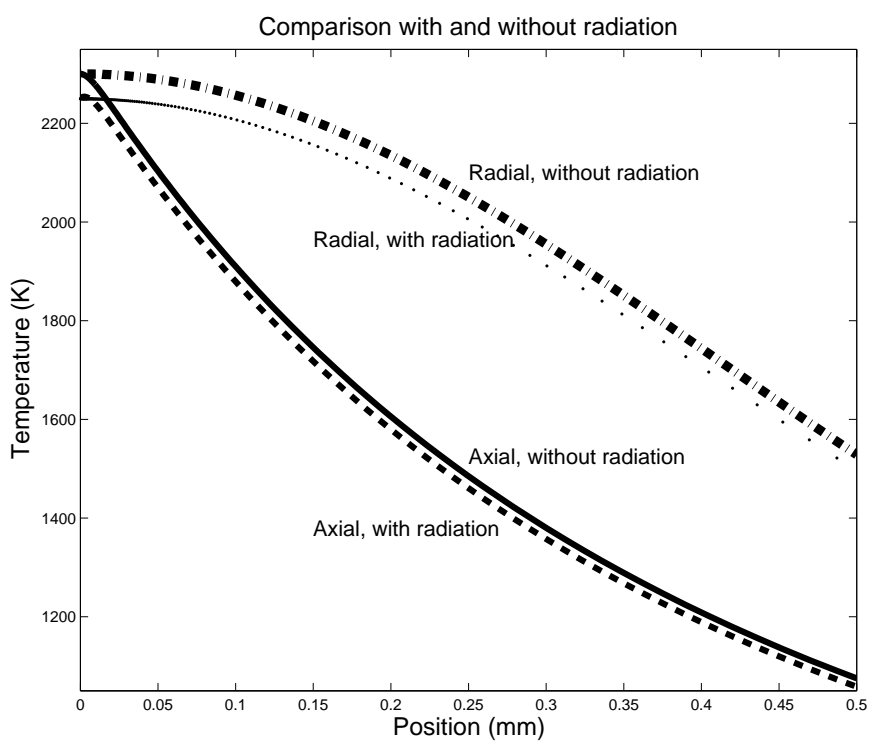

Figure 8: Axial and radial temperature profiles. 2D simulation at end of a $10 \mathrm{~s}$ exposure using $10 \mathrm{~W}$ total laser power and a $1 \mathrm{~mm}(1 / \mathrm{e})$ beam diameter.

the same reasons described above. For the case of strong (local laser energy) absorption considered here, the effect of radiation is to uniformly cool the region resulting in a near constant temperature difference, both radially and axially. In the case considered, most of the energy is absorbed near the surface. In comparison to a simulation using only conduction, radiation effectively reduces the energy absorbed by the material essentially scaling the resulting temperature field. For more weakly absorbing lasers, radiation transport would affect the shape as well as the magnitude of the temperature distribution.

\section{Conclusion}

We have derived a system of equations that simulate radiative transport in refractive lossy media. The equations are implemented in a widely used code that up to now, even though it contained hydrodynamic, material strength, MHD and heat conduction models, lacked a radiation package. Since the code is used in relatively low temperatures regimes, radiative effects were thought to be negligible since the energy content in radiation was overwhelmed by the energy of the medium. (Radiation energy density scales as $a T_{r}^{4}$, where $a=7.6 \cdot 10^{-15}$ $\mathrm{erg} / \mathrm{cm}^{3}{ }^{\circ} \mathrm{K}^{4}$.) However, as shown in the simulations, although there is little energy in radiation, it is an efficient vehicle for transporting energy. Indeed, if the hot slab described in Section 6.1 was instead surrounded by vacuum, the 
only mechanism for heat loss is radiation.

We conclude with several observations and reservations about our model. First, in contrast with other low temperature applications with radiation, we keep the temporal derivative $\partial_{t} E$. The term is indeed small in comparison with terms such as the change of internal energy $C \partial_{t} T$, radiation absorption $\kappa v_{g} E$ and diffusion of radiation flux $\nabla \cdot\left(v_{g} / 3 \kappa\right) \nabla E$. But keeping $\partial_{t} E$ does no harm nor does it complicate the numerics. Although we discretize using finite elements, we use a lumped mass matrix for $\partial_{t} E$. The term brings extra stabilization by adding to the diagonal of the resulting linear system. Furthermore, for large $\Delta t$ our fully implicit differencing recovers the hops from one steady state to another inherent in models that neglect $\partial_{t} E$.

For small $\Delta t$, the term $\partial_{t} E$ enables tracking changes to the $E$ field, as shown in the decay of $T_{r}$ at $t=10^{-10}$ in the problem discussed in Section 6.1. Since we model transport with diffusion, to avoid energy propagating at unphysically large speeds, we should modify the diffusion coefficient $v_{g} / 3 \kappa$ with a flux limiter. Although we will do that, we have encountered mysterious behavior after comparing results from simulations using different radiation transport models. We compared our results with those from a conventional rad-hydro code (no reflection, no refraction.) The slab appears to be optically thin since quickly, with diffusion too quickly, radiation establishes a quasi steady state characterized by bulk emission and energy loss at the boundary. With $S_{n}$ transport, a cooling wave propagates inward with a speed approximately equal to $c$. At $t=10^{-12}$, the wave's leading edge has penetrated $0.7-0.8 \mathrm{~mm}$, which given the coarse discretization $\Delta Z=0.1 \mathrm{~mm}$, is reasonably close to the causal distance $0.3 \mathrm{~mm}$. In front of the wave, $T_{r}$ is in its initial state, $2500{ }^{\circ} \mathrm{K}$. However, with flux-limited diffusion, by $t=10^{-12}$, the wave has already reached the midplane $(Z=5 \mathrm{~mm})$ since $\max \left(T_{r}\right)$ has decreased by $2-3{ }^{\circ} \mathrm{K}$. The mystery is that we obtain the same result without the flux limiter. For this problem, surprisingly, the limiter has no effect. Although we will investigate this further, we stress that for the relatively large time steps used in annealing simulations, one rarely models temporal changes of $E$; the flux limiter is needed only when $\partial_{t} E$ matters. Nevertheless, it bears repeating that for transparent media, mean free paths are, by definition, long; hence, the diffusion approximation is not expected to be a good model of transport.

Our final reservation regards the coefficients $n$ and $\kappa$. Our derivation assumes a homogeneous medium, i.e., $n$ and $\kappa$ are independent of time and space. In reality, though, they're not. At room temperature glass is transparent. However, any visitor to a glass blowing factory can attest that when the sample is removed from the furnace, it glows, i.e., it is no longer transparent. In the optical range, the opacity has surely increased by orders of magnitude. Our derivation ignores this. However, we are currently hampered by a paucity of data. Although there is scattered data for the variation of the absorption index $k$ with $T$, e.g., McLachlan [15], at this time we are unaware of the $k(T)$ variation for all frequencies. A related concern is that a full description of $k(T)$ is 
insufficient. Because of the Kramers-Krönig relations, Jackson [8], Kitamura et al [10], a change in $k$ affects $n$. We conclude by recalling the equations derived by Pomraning [17] for a medium with spatially and temporally varying $n$. In such materials, transport is extremely complicated; as they traverse such media, photons follow curved, not straight, paths. And they change color! There is clearly more work to do.

Lastly, we note that although it's long been recognized that radiation plays an important role in thermal transport in semi-transparent media, it can apparently be ignored in certain applications. Recent work by Yang et al [23] show that one obtains relatively good comparison with experiment by, surprisingly, using only linear heat conduction with constant material properties.

\section{Acknowledgement}

This work was supported by the Lawrence Livermore National Laboratory directed research program, contract LDRD:08-ERD-057.

\section{References}

[1] M. Born and E. Wolf, Principles of Optics, Pergammon Press, OxFord (1975)

[2] P. Bouchut, D. Decruppe and L. Delrive, "Fused silica thermal conductivity dispersion at high temperature," J. Appl. Phys., 96, 6, 3221-3227 (2004)

[3] J. I. Castor, Radiation Hydrodynamics, Cambridge Press, Cambridge (ISBN 052183309 4) (2004)

[4] S. W. Churchill and H. H. S. Chu, "Correlating equations for laminar and turbulent free convection from a vertical plate," Int. J. Heat Mass Transfer," 18, 1323-1329 (1975)

[5] E. U. Condon, "Radiative transport in hot glass," J. Quant. Spectrosc. Radiat. Transfer," 28, 369-385 (1968)

[6] http://www.engineeringtoolbox.com/convective-heat-transfer-d_430.html

[7] R. Gardon, "A review of radiant heat transfer in glass," J. Amer. Ceramic Soc., 44, 7, 305-312 (1961)

[8] J. D. Jackson, "Classical Electrodynamics Second Edition," J. Wiley \& Sons, Inc., New York (1975)

[9] J. O. Isard, "Surface reflectivity of strongly absorbing media and calculation of the infrared emissivity of glasses," Infrared Phys., 20, 249-256 (1980) 
[10] R. Kitamura, L. Pilon and M. Jonasz, "Optical constants of silica glass from extreme ultraviolet to far infrared at near room temperature," Applied Optics, 46, 33, 8118-8133 (2007)

[11] E. W. Larsen, G. Thömmes, A. Klar, M. Seaid and T. Götz, "Simplified $P_{N}$ Approximations to the Equations of Radiative Heat Transfer and Applications," J. Comp. Phys., 183, 652-675 (2002)

[12] E. W. Larsen, G. Thömmes and A. Klar, "New Frequency-Averaged Approximations to the Equations of Radiative Heat Transfer," SIAM J. Appl. Math., 164, 2, 565-582 (2003)

[13] E. W. Larsen, J. E. Morel and J. M. McGhee, "Asymptotic derivation of the multigroup $P_{1}$ and simplified $P_{n}$ equations with anisotropic scattering," Nuc. Sci. Eng., 123, 328 (1996)

[14] C. M. Lund and J. R. Wilson, "Some Numerical Methods for TimeDependent Multifrequency Radiation Transport Calculations," Lawrence Livermore Natl. Lab. report UCRL-84678, July 29, (1980)

[15] A. D. McLachlan and F. P. Meyer, "Temperature dependence of the extinction coefficient of fused silica for $\mathrm{CO}_{2}$ laser wavelengths," Applied Optics, 26, 9, 1728-1731 (1987)

[16] L. Martí-López, J. Bouza-Domíguez, R. A. Martínez-Celorio and J. C. Hebden, "An investigation of the ability of modified radiative transfer equations to accommodate laws of geometrical optics," Optics Com., 266, 44-49 (2006)

[17] G. C. Pomraning, The Equations of Radiation Hydrodynamics, Dover Pub. Inc., Mineola, New York (2005)

[18] G. C. Pomraning, A. K. Prinja and I. R. Shokair, "On the use of mean absorption coefficients in the presence of strong temperature gradients," $J$. Quant. Spectrosc. Radiat. Transfer," 26, 3, 199-213 (1981)

[19] A. I. Shestakov, J. A. Harte and D. S. Kershaw, "Solution of the Diffusion Equation by Finite Elements in Lagrangian Hydrodynamic Codes," J. Comp. Phys., 76, 2 (1988)

[20] A. I. Shestakov, "Conversion of Larsen's "Partially Averaged $S P_{2}$ equations to conventional multigroup form," Lawrence Livermore Natl. Lab. internal report (2009)

[21] J. S. Stölken, private communication, Lawrence Livermore National Laboratory (2009)

[22] http://www.tpdsci.com/Tpc/RIQtzFsd.php and http://repositories.cdlib. org/escholarship 
[23] S. T. Yang, M. J. Matthews, S. Elhadj, V. G. Dragoo, and S. E. Bison, "Thermal transport in $\mathrm{CO}_{2}$ laser irradiated fused silica: In situ measurements and analysis, J. Appl. Physics, 106, 103106 (2009)

[24] Ya. B. Zel'dovich and Yu. P. Raizer, Physics of Shock Waves and HighTemperature Hydrodynamic Phenomena, Dover, New York 0-486-42002-7 (2001) 